\title{
Übernimmt die Autobranche Vertrieb und Netzmanagement der E-Wirtschaft?
}

\author{
M.-V. Fischer \\ Online publiziert am 14. Februar 2017 \\ (c) Springer Verlag Wien 2017
}

Durch die Elektrifizierung und Digitalisierung steigen die Bedrohungsszenarien für die Autobranche: Mit Apple, Google, Tesla und Co. drängen immer mehr Konkurrenten in den Markt, durch autonomes Fahren können weniger Emotionen beim Kauf vermittelt werden, und Carsharing reduziert den Bedarf an Fahrzeugen pro Person.

Die Geschäftsmodelle der Hersteller stehen daher unter zunehmendem Druck. Die OEMs müssen sich deshalb neue Geschäftsmodelle suchen.

Eines der zentralen Zielfelder hierbei ist die Energiewirtschaft. BMW verkauft schon heute in Deutschland zum elektrischen i3 Na- turstromverträge und Carports samt PV-Anlage. Auch Tesla ist bereits in die Photovoltaikbranche eingestiegen und bietet stationäre Stromspeicher an. Die Konsequenz für Energieversorger ist düster mit dem Stromkunden gehen auch die Adresse und damit einhergehende Upselling-Möglichkeiten verloren. Auch beim Thema Vehicleto-grid versuchen sich die Autohersteller als Gatekeeper. Die Kundenadresse wird zum Öl der Zukunft, sonst ist der Energieversorger nur mehr Sublieferant!

Kurzfassung eines Vortrags bei der 54. Fachtagung der Österreichischen Gesellschaft für Energietechnik (OGE) im OVE, die am 24. und 25. November 2016 in Villach stattfand.

Fischer, Michael-Viktor,

SMATRICS GmbH \& Co KG, Europaplatz 2/4/3, 1150 Wien, Österreich

E-Mail: michael.fischer@smatrics.com 